\title{
An optimization-based control strategy for energy efficiency of discrete manufacturing systems
}

\author{
Jenny L. Diaz ${ }^{\mathrm{a}, *}$, Miguel Bermeo ${ }^{\mathrm{a}}$, Javier Diaz-Rozo ${ }^{\mathrm{b}}$, Carlos Ocampo-Martinez $^{\mathrm{a}}$ \\ ${ }^{a}$ Automatic Control Department, Universitat Politècnica de Catalunya, Institut de Robòtica i Informàtica Industrial (CSIC-UPC), Llorens i Artigas 4-6, Planta 2, \\ 08028 Barcelona, Spain. \\ ${ }^{b}$ Ikergune AIE, San Antolin 3, Elgoibar 20870, Spain.
}

\begin{abstract}
In order to reduce the global energy consumption and avoid highest power peaks during operation of manufacturing systems, an optimization-based controller for selective switching on/off of peripheral devices in a test bench that emulates the energy consumption of a periodic system is proposed. First, energy consumption models for the test-bench devices are obtained based on data and subspace identification methods. Next, a control strategy is designed based on both optimization and receding horizon approach, considering the energy consumption models, operating constraints, and the real processes performed by peripheral devices. Thus, a control policy based on dynamical models of peripheral devices is proposed to reduce the energy consumption of the manufacturing systems without sacrificing the productivity. Afterward, the proposed strategy is validated in the test bench and comparing to a typical rule-based control scheme commonly used for these manufacturing systems. Based on the obtained results, reductions near $7 \%$ could be achieved allowing improvements in energy efficiency via minimization of the energy costs related to nominal power purchased.
\end{abstract}

Keywords: Energy management, discrete manufacturing systems, optimization-based control, model predictive control

\section{Introduction}

Manufacturing industry accounts for 50\% of energy consumed by the industrial sector, which corresponds to $30 \%$ of the electrical energy produced around the world [1]. Thus, factors such as the depletion of fossil energy sources, the increasing energy prices, and the governmental regulation have imposed new challenges to industry. From this fact, manufacturing industry has focused on looking for strategies and technologies that allow improving its energy efficiency by reducing energy costs and optimizing the use of that energy and related resources. Thereby, both the economic and environmental dimensions have gained attention during the last decade. Regarding the environmental dimension, strategies have focussed on proposing new designs for machine devices, the use of technologies and resources more environmental friendly, energy supply from renewable sources, cogeneration plants for in situ electric power generation, among others [2, 3]. In addition, the economic dimension has been deeply studied due to its direct effect over the energy costs. In this way, several strategies, which include improvements in technologies for data acquisition and analysis, as well as for modeling, monitoring, and control of manufacturing systems, have been proposed $[4,5,6]$.

In this context, manufacturing systems are understood as arrangements of different devices that work in a periodic, coordinated and sequential manner, e.g., a machine for manufacturing a piece. Thus, these devices can be classified into those

\footnotetext{
${ }^{*}$ Corresponding author

Email address: jdiaz@iri.upc.edu (Jenny L. Diaz )
}

directly involved into the manufacturing processes (e.g., forming, machining, joining), and those that guarantee the operating conditions of these processes without their direct participation. These latter are known as peripheral devices and, in a process line, could be shared by two or more machines or manufacturing systems. According to this classification, most of the strategies implemented so far are mainly oriented to reduce the idle times and the total energy consumption through an off-line optimization of a particular manufacturing sequence, i.e., process planing and scheduling [7]. These approaches are essential and complementary factors regarding energy consumption and flexibility of manufacturing systems. Thus, the problem of process planning and scheduling has been usually formulated as a multi-objective optimization problem that considers both the makespan and the energy consumption of a machine cycle $[8,9]$. However, most of the applications of this topic have been mainly oriented to both process line and plant levels.

In addition to the global energy consumption in a fixed period, manufacturing systems can show additional energy costs when their energy consumption exceeds a maximum contracted power, fact that could occur due to the simultaneous activation of several devices, yielding in undesirable power peaks. Therefore, although the off-line optimization of the activation sequence of manufacturing devices (including or not the peripheral devices) allows improving the energy efficiency of a manufacturing system by minimizing its global energy consumption, other concerns must be taken into account to develop strategies that can be successful in real time and when disturbances take place. In this sense, strategies that avoid surpassing the nomi- 
nal contracted power, e.g., by using selective on/off switching of the peripheral devices [10], could be considered.

Some proposals for solving in real time the energy efficiency issue include the design of control systems, from which the peripheral devices can be managed taking into account the energy consumption of the whole set of devices. In this case, optimization-based control (OBC) techniques have had a great application due to their high customization level for defining control objectives, and including operating constraints of both manufacturing and peripheral devices into the controller design. Likewise, due to the complexity of manufacturing systems, most of the models used for the design of control strategies are based on input-output correlations from data sets of energy consumption. This latter fact is given since the physicalbased models require the full knowledge of several physical dynamics and parameters, which are often hard to represent, compute or estimate [5, 11, 4].

As a way to overcome the drawback of requiring physicalbased models, the process models obtained from subspace identification (SI) methods have gained attention in manufacturing systems management, since these methods directly deliver a state-space model of less complex implementation for the design of model-based control strategies [12]. As a consequence, SI models have had significant application to the modeling of complex, large-scale, and time-varying-parameter systems $[13,14,15]$. Then, due to the applicability of SI for obtaining models to be used in model-based control design (in particular for model predictive control - MPC - design), they have been widely used in manufacturing industries [16, 17].

Based on the previous discussion, the main contribution of this paper is focused on proposing an energy reduction control approach for peak-power suppression in real time, which is applied to periodic manufacturing systems in which their peripheral devices can be independently managed. The proposed control strategy is based on power consumption models of both the manufacturing system and its peripheral devices, which have been determined by using SI methods. Then, the proposed controller is designed based on OBC techniques and the receding horizon philosophy in order to formulate an optimization problem that includes operating constraints and relationships among the manufacturing and peripheral devices. Thus, the aim is to predict the time instant at which peripheral devices will be required and, based on this prediction, selecting the time instant in which those devices must be switched on/off. This last fact with the aim to avoid surpassing the purchased nominal power, besides of guaranteeing the time-varying operating conditions of manufacturing processes and without sacrificing their productivity. Then, in order to show the effectiveness of the proposed approach, a case study based on a real test bench that emulates the energy consumption of both manufacturing processes and peripheral devices is developed.

The remainder of the paper is organized as follows. In Section 2, the statement of the problem considered in this paper is presented. Next, Section 3 presents and discusses the proposed control approach, including the way the energy consumption models have been obtained by using SI methods. In Section 4, the considered case study and a description of the test bench

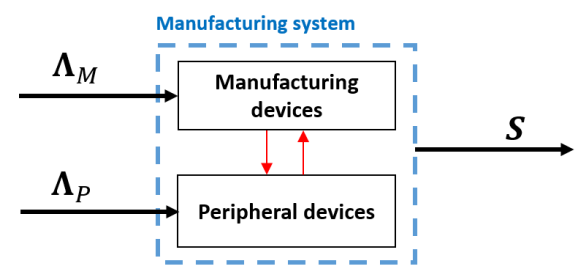

Figure 1: General scheme of inputs and outputs of machine tool.

setup are presented. Afterwards, obtained results from the implementation of the proposed approach in the test bench are reported and discussed in Section 5. Finally, in Section 6, conclusions and future works based on the obtained results are drawn.

\section{Problem Statement}

A discrete manufacturing system (e.g. a machine) can be considered as a set of different devices that work in a sequential way to process a piece during a fixed period of time. In this way, a periodic behavior characterizes these systems according to the total time required for manufacturing a piece, which corresponds to a operation cycle denoted here by $T$. Thus, the energy consumption of devices straight related to manufacturing operations, such as manufacturing processes, transport, and handle of pieces, shows also a periodic behavior. In addition to the devices directly involved in manufacturing operations, there are peripheral devices that guarantee the supply of resources (e.g., comprised air, water, coolant, lubricants) to the main devices, and which might or might not show a periodic behavior, which may match with $T$. Therefore, due to the nature of the operations performed in manufacturing systems (e.g., transport, rotational motions, axial motions, cutting, milling), there exist stages (or modes) of both high and low energy consumption along $T$.

Based on the stages of both higher and lower energy consumptions along $T$, peripheral devices must be correctly managed such that their activation time does not match with the time instants/slots of higher consumption of the manufacturing operations, avoiding also (if possible) the simultaneous activation of peripheral devices. Besides, the activation times of each peripheral device should be selected taking into account both its operating constraints and the dependency on the operation cycle. A manufacturing system and its associated set of peripheral devices can be represented as shown in Fig. 1, being $\boldsymbol{\Lambda}_{\mathbf{M}}$ and $\boldsymbol{\Lambda}_{\mathbf{P}}$ the activation sequences of manufacturing and peripheral devices, respectively, while $S$ is the apparent power consumed by the entire system.

Thus, considering a fixed number of both manufacturing and peripheral devices related to a single ${ }^{1}$ machine or system, the activation sequences can be defined as follows:

$$
\begin{aligned}
\boldsymbol{\Lambda}_{\mathbf{M}}(k) & =\left\{u_{M_{1}}(k), u_{M_{2}}(k), \ldots, u_{M_{m}}(k)\right\}, \\
\boldsymbol{\Lambda}_{\mathbf{P}}(k) & =\left\{u_{P_{1}}(k), u_{P_{2}}(k), \ldots, u_{P_{n}}(k)\right\},
\end{aligned}
$$

\footnotetext{
${ }^{1}$ Without lost of generality, this notation represents the case of non-shared peripheral devices. The extension is straightforward.
} 
being $k \in \mathbb{Z}_{\geq 0}$ the discrete-time index and $m=\left|\boldsymbol{\Lambda}_{\mathbf{M}}\right|$ and $n=\left|\boldsymbol{\Lambda}_{\mathbf{P}}\right|$ the number of manufacturing and peripheral devices, respectively. Besides, $u_{M_{j}}(k) \in\{0,1\}, l \in \mathcal{L} \triangleq\{1,2, \cdots, m\}$, and $u_{P_{j}}(k) \in\{0,1\}, j \in \mathcal{J} \triangleq\{1,2, \cdots, n\}$ are the activation times of the $l$-th manufacturing device and the $j$-th peripheral device, respectively. However, it should be noted that in cases in which the load of the peripheral devices can be modulated, $u_{P_{j}} \in \mathbb{Z}_{\geq 0}$.

For the case of manufacturing devices, the execution times $T_{M_{l}}$ are usually fixed and their operation is constrained into $T$ (with $T$ as the upper bound). Therefore, $\sum_{l=1}^{m} T_{M_{l}}=T$ holds when only one manufacturing device is turned on and only once during $T$. On the other hand, since the peripheral devices might not show a periodic behavior, their operation is not constrained into $T$ and their execution times $T_{P_{j}}$ are not necessarily upper bounded by $T$. Thus, given the periodicity of $\boldsymbol{\Lambda}_{\mathbf{M}}$, its apparent power consumption, namely $S_{M}$, can be considered as fixed and periodic. Consequently, both $u_{M_{l}}(k)$ and $T_{M_{l}}$ are given by the manufacturing process and are known a priori. In this sense, Assumption 1 is established.

Assumption 1. The manufacturing sequence $\boldsymbol{\Lambda}_{M}$ is given and hence its associated apparent power consumption, denoted by $\bar{\beta} \triangleq \sum_{k=0}^{T} S_{M}\left(\boldsymbol{\Lambda}_{\mathbf{M}}(k)\right)$, when a periodic behavior is considered.

On the other hand, the energy consumption from peripheral devices depends on the operational relationships between both manufacturing and peripheral devices, which are needed to guarantee the operating conditions of the whole manufacturing processes. Thereby, in order to select the optimal activation instants of the peripheral devices regarding the global energy consumption, the dynamics of both energy consumption and the process itself, and operating constraints of these devices must be considered into the problem formulation because of the settling time of each element. In this sense, the control problem consists in determining the optimal $\boldsymbol{\Lambda}_{\mathbf{P}}$ that minimizes both the global energy consumption $S$ and the peaks of $S$ that could exceed the nominal power purchased along a fixed period, e.g., $T$. Indeed, considering a operation time with length $T$, the control objective can be defined as to minimize the energy consumption profile of peripheral devices according to the instantaneous energy consumption of $\boldsymbol{\Lambda}_{\mathbf{M}}$ along $T$, i.e.,

$$
J=\sum_{k=1}^{k=T} \bar{\beta}(k) S_{P}\left(k, \boldsymbol{\Lambda}_{\mathbf{P}}(k)\right),
$$

being $\bar{\beta}(k) \in \mathbb{R}_{\geq 0}$ the apparent power consumption related to $\boldsymbol{\Lambda}_{\mathbf{M}}$ at each $k \in \mathbb{Z}_{\geq 0}$, with $S_{P} \in \mathbb{R}_{\geq 0}$.

Therefore, in order to achieve the control objective, activation/deactivation of peripheral devices $j \in \mathcal{J}$ must be suitably managed subject to their operating constraints. Besides, in order to compute the global apparent power consumption $S(k)=S_{P}(k)+\bar{\beta}(k)$, apparent power consumption models for each peripheral device are required, i.e.,

$$
S_{P_{j}}(k)=f_{j}\left(\xi(k), u_{P_{j}}(k)\right),
$$

being $\xi(k)$ the states of the energy consumption model, and where $f_{j}:\{0,1\} \mapsto \mathbb{R}_{\geq 0}$ is, in general, a nonlinear map in function of the individual input signal that activates/deactivates the $j$-th device. Hence, $S_{P}\left(k, \Lambda_{\mathbf{P}}(k)\right) \approx \sum_{j=1}^{n} S_{P_{j}}(k)$.

Moreover, there exist several relationships among $\boldsymbol{\Lambda}_{M}$ and $\boldsymbol{\Lambda}_{P}$, which determine the proper behavior of the peripheral devices, e.g., the supply lubricant fluids from a central deposit through a pump, air from a compressor, chip transport, among others. These relations, which could be of dynamic nature, can be defined as

$$
q_{r}(k+1)=g_{r}\left(v, \boldsymbol{\Lambda}_{\mathbf{P}}(k)\right),
$$

for $r \in \mathcal{Q} \triangleq\{1,2, \cdots, Q\}$, where $Q$ is the number of existing relations $q_{r}$ and $v=h\left(\boldsymbol{\Lambda}_{\mathbf{M}}\right) \in \mathbb{R}$ is the particular relationship between $q_{r}$ and $\boldsymbol{\Lambda}_{\mathbf{M}}$. Besides, $h:\{0,1\} \times \mathbb{R} \mapsto \mathbb{R}$ and $g_{r}: \mathbb{R}^{m} \times$ $\{0,1\}^{n} \mapsto \mathbb{R}$ are the maps that define such relations $q_{r}$. Notice that, in this case, $q_{r}$ is considered as some process variable of a particular peripheral device, which is directly related to $\boldsymbol{\Lambda}_{\mathbf{M}}$.

\section{Proposed approach}

In order to improve energy efficiency during the operation of manufacturing systems and its peripheral devices, the main contribution of this paper will be developed throughout this section. In this regard, an optimization-based controller is proposed considering both energy consumption models and operating constraints of peripheral devices into an optimization problem behind the design of such a controller. Thus, the general idea is to use the receding horizon approach to anticipate either activation or deactivation of peripheral devices taking into account their dynamics and the global energy consumption $S$. Therefore, a control policy for the selective switching on/off of peripheral devices according to their dynamics is established in order to reduce the global energy consumption. It should be noted that, as shown below, the proposed control policy does not affect the system productivity since the machining sequence is fixed while only activation instants of the peripheral devices are changed. In this way, the operating cycle of the machine remains the same.

Given the consideration of a prediction horizon $H_{p}$, the decision of switching on or off a device $j \in \mathcal{J}$ depends on the current value of $\beta(k)$, i.e., although the manufacturing sequence is already given and hence its energy consumption, its consumption values discriminated along the time are important for making decisions regarding peripheral devices management. According to the control objective defined in (2), the sequences ${ }^{2}$ for $J$ and $\boldsymbol{\Lambda}_{\mathbf{P}}$ along $H_{p}$ are defined as

$$
\begin{aligned}
& \mathbf{J}(k) \triangleq\left\{J(k \mid k), \ldots J\left(k+H_{p}-1 \mid k\right)\right\}, \\
& \boldsymbol{\Gamma}(k) \triangleq\left\{\boldsymbol{\Lambda}_{\mathbf{P}}(k \mid k), \ldots, \boldsymbol{\Lambda}_{\mathbf{P}}\left(k+H_{p}-1 \mid k\right)\right\},
\end{aligned}
$$

with $\mathbf{J}(k) \in \mathbb{R}^{H_{p}}, \boldsymbol{\Gamma}(k) \in\{0,1\}^{n H_{p}}$. Besides, the polytopic constraint of peripheral devices is represent by

$$
\mathbb{Q}=\left\{q_{r} \in \mathbb{R}^{r} \mid q_{r}(k) \in\left[\underline{q}_{r}, \bar{q}_{r}\right] \forall k,\left\{\underline{q}_{r}, \bar{q}_{r}\right\} \in \mathbb{R}\right\} .
$$

\footnotetext{
${ }^{2}$ Here, $z(k+i \mid k)$ denotes the prediction over $H_{p}$ of the variable $z$ at time instant $k+i$ performed at $k$.
} 
Thus, the design of the proposed predictive-like controller is based on the following finite-time open-loop optimization problem:

$$
\min _{\Gamma(\mathbf{k})} \mathbf{J}(k)
$$

subject to

$$
\begin{aligned}
q_{r}(k+i+1 \mid k) & =g_{r}\left(v, \boldsymbol{\Lambda}_{P}(k+i \mid k)\right), \\
S_{P_{j}}(k+i \mid k) & =f_{j}\left(\xi(k+i \mid k), u_{P_{j}}(k+i \mid k)\right), \\
u_{P_{j}}(k+i \mid k) & \in\{0,1\} \cup \mathbb{Z}, \\
q_{r}(k+i \mid k) & \in \mathbb{Q},
\end{aligned}
$$

for $i \in\left\{0,1,2, . ., H_{p}-1\right\}, j \in \mathcal{J}, r \in \mathcal{Q}$.

Assuming that the problem (7) is feasible, i.e., $\boldsymbol{\Gamma}(k) \neq \emptyset$, there will be an optimal solution for the activation sequence of peripheral devices defined by

$$
\boldsymbol{\Gamma}^{*}(k) \triangleq\left\{\boldsymbol{\Lambda}_{\mathbf{P}}{ }^{*}(k \mid k), \ldots, \boldsymbol{\Lambda}_{\mathbf{P}}{ }^{*}\left(k+H_{p}-1 \mid k\right)\right\},
$$

and then, according to the receding horizon philosophy [18, 19], $\boldsymbol{\Lambda}_{\mathbf{P}}{ }^{*}(k \mid k)$ is applied to the system discarding the rest of the optimal sequence from $(k+1) \mid k$ to $\left(k+H_{p}-1\right) \mid k$, while the whole process is repeated for the next time instant $k \in \mathbb{Z}_{\geq 0}$ after measuring/estimating the proper information from the plant to be used as the update for the energy consumption models considered in (7c).

Notice that the optimization problem (7) explicitly considers both the consumption models and the operating constraints of the peripheral devices ((7c) and (7b), respectively). Therefore, suitable expressions for the maps $f_{j}$ and $g_{r}$ should be proposed according to the real operation of the peripheral devices. Given the possible nonlinear nature of maps $f_{j}$, this paper proposes the identification of input-output models based on the SI methods. Thus, from a proper set of input-output data, i.e., activation sequences $\boldsymbol{\Lambda}_{\mathbf{P}}$ and apparent power $S_{P}$, such models can be obtained.

On the other hand, regarding maps $g_{r}$, dynamics expressions that consider the dependency on $\boldsymbol{\Lambda}_{\mathbf{M}}$ are proposed taking into account the processes performed by peripheral devices. Therefore, the proposal for modeling energy consumption and $q$-relations for peripheral devices, and the design of the optimization-based controller towards reaching the control objective while satisfying all the physical and operational constraints stated are introduced in the next sections.

\subsection{Model identification}

Different approaches have been addressed for modeling and designing control strategies in manufacturing systems. Among others, the Markov chains (MC) and Petri Nets (PN) can be highlighted given their great application at both process line and plant levels [20]. The main applications of these approaches have been oriented to model flow lines with a serial movement of jobs among workstations, job shops with more flexibility, assembly lines, among others, which serve as a foundation for design, scheduling, and control. Some applications and deeper explanations about these modeling approaches can be found in
$[21,22,23]$. Despite the great application of both MC and PN in the manufacturing systems, they have been focused on modeling the production states of the machine rather than modeling the energy consumption behavior of such systems. Thus, since the complexity to establish the different machine states, transitions between them, and their connections, data-driven models, such as those obtained from the SI methods, have gained attention into the manufacturing industry to model its energy consumption.

Thereby, for determining the power consumption models of both manufacturing and peripheral devices, SI methods are introduced in this section. Based on real input-output data of a system, SI methods allow the identification of matrices of a state space representation for Linear Time-Invariant (LTI) systems. Thus, for a given set of input-output measurements of length $d$, with $b \geq 1$ input signals and $p \geq 1$ output signals resulting from feeding a dynamic system with such inputs, the SI problem consists of [24]

(a) estimate the system order $N$,

(b) estimate the systems matrices $A \in \mathbb{R}^{\ell \times \ell}, B \in \mathbb{R}^{\ell \times b}, C \in$ $\mathbb{R}^{p \times \ell}$, and $D \in \mathbb{R}^{p \times b}$,

for which, matrices $A, B, C, D$ satisfy a state-space (unknown) realization of order $N$, i.e.,

$$
\begin{aligned}
x(k+1) & =A x(k)+B u(k)+w(k), \\
y(k) & =C x(k)+D u(k)+v(k),
\end{aligned}
$$

with $x \in \mathbb{R}^{\ell}, u \in \mathbb{R}^{b}$ and $y \in \mathbb{R}^{p}$ the state, input and output vectors, respectively, while $w \in \mathbb{R}^{\ell}$ and $v \in \mathbb{R}^{p}$ are the state noise and output measurement noise, respectively.

For determining model matrices and $N$, this paper focuses on the Numerical algorithms For Subspace IDentification (N4SID) [25] because of its great application and implementation in software. The N4SID algorithms first estimate the state $\hat{x}$ from the projection of input-output data, and then, systems matrices are determined based on the estimated state sequence. A detailed review about the different SI algorithms and their implementation can be found in $[12,24,26,25]$. Thereby, different activation sequences $\boldsymbol{\Lambda}_{\mathbf{P}}$ (as inputs) should be evaluated to consider the most of possible scenarios and get information about their apparent power $S_{P}$ consumed (as outputs).

\subsection{Operation of peripheral devices}

Peripheral devices of manufacturing systems perform different processes to supply the necessary resources for the proper operation of manufacturing devices, e.g., compressed air, lubricant, coolant. Mathematical expressions for defining relations between $\boldsymbol{\Lambda}_{\mathbf{M}}$ and $\boldsymbol{\Lambda}_{\mathbf{P}}$ should be established considering both the resources consumption from a manufacturing system and the process performed by the peripheral device.

According to different manufacturing systems, some peripheral devices can or cannot be critical for the manufacturing processes, and in this sense, some of these devices could be controlled either independently or dependently. Besides, some peripheral devices can have a buffer capacity, which could be enough to cover the whole operation cycle or even more. Thus, 
based on the analyzed system, peripheral devices with different functionalities and capacities can be found. Due to this fact, three criteria are considered to establish the priorities for managing peripheral devices and to define the $q_{r}$-relations to be used in the optimization problem. The factors considered are:

1. Safety of manufacturing process: A peripheral device is considered critical for the safety of processes if that device directly supplies one resource necessary for the manufacturing process, e.g., compressed air, coolant, lubricant. An example of those devices/processes is the coolant feeding, which deliveries the coolant required for machining a piece in a machine tool.

2. Buffer capacity: Based on the particular design of peripheral devices, some peripheral devices could have capacity enough to cover the whole operation cycle without a realtime energy conversion during their operations. In addition, other devices may not have buffering capacity or not enough to cover a operation cycle, which implies that these devices perform their processes based on real-time energy conversion. Some example of these buffers are the pressure reservoirs, tanks, trays, among others.

3. Control depending on main manufacturing processes: Based on the relationships between manufacturing and peripheral devices, these latter could be controlled according to the manufacturing processes when some event directly related to their operation triggered the activation/deactivation of peripheral devices. On the other hand, some devices can be controlled based on time and independent on manufacturing process, indeed, their switching on/off is allowed only in fixed time intervals.

Based on these factors, it is possible to identify the devices that require a real-time energy conversion during their operation and those devices able to satisfy the requirements of manufacturing process without being turned on at the same time. However, additional to these relations as constraints in the optimization problem, there exist other operating limitations of peripheral devices, such as idle times, running times, and switching frequency, etc., that should be considered.

\subsection{Control strategy of energy consumption}

From the optimization problem formulated in (7), in this paper a predictive-like controller based on the receding horizon approach and a linear mixed-integer model is proposed. According to the power consumption models, the $q$-relations, and the operating limitations of peripheral devices, the proposed control scheme to find $\Gamma^{*}(k \mid k)$ along $H_{p}$ is presented in Figure 2.

In the proposed closed-loop control scheme, the optimization problem in (7) is solved into a controller module. Afterward, once an optimal $\boldsymbol{\Gamma}^{*}(k \mid k)$ is determined, only the first component $\boldsymbol{\Lambda}_{\mathbf{P}}{ }^{*}(k \mid k)$ is applied to the plant. The observer module receives at each sampling time $k$ the power measurement and the current system inputs and estimates the current state of the plant, which is fed back to the controller.

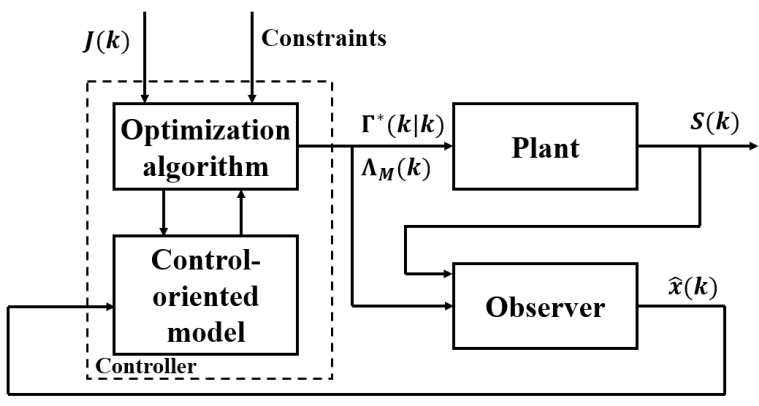

Figure 2: Control scheme of energy consumption in a machine tool.

Observer module: Since the system is composed of the individual power consumption models for both the manufacturing and peripheral devices, the total output $S$ can be defined as the sum of $S_{M}$ and $S_{P}$, assuming there is no energy correlation between them. Thus, previously to the design of the observer module, a total power consumption model is defined by extending the model matrices as follows:

$$
A_{e}=\left[\begin{array}{cccc}
A_{M} & 0 & \cdots & 0 \\
0 & A_{P_{1}} & \cdots & 0 \\
\vdots & \vdots & \ddots & \vdots \\
0 & 0 & 0 & A_{P_{j}}
\end{array}\right],
$$

and, similarly, for $B_{e}, C_{e}$, and $D_{e}$. Next, considering $u(k)=\left[u_{M}(k), u_{P_{1}}(k), \ldots, u_{P_{j}}(k)\right]^{T}$ and $x(k)=$ $\left[x_{M}(k), x_{P_{1}}(k), \ldots, x_{P_{j}}(k)\right]^{T}$ being the extended input and state vectors, respectively, a Kalman filter to determine the estimation of the states of both manufacturing and peripheral devices from the overall system output $S$ is designed as follows:

$$
\begin{aligned}
\hat{x}(k+1) & =A_{e} \hat{x}(k)+B_{e} u(k)+L(S(k)-\hat{S}(k)), \\
\hat{S}(k) & =C_{e} \hat{x}(k)+D_{e} u(k),
\end{aligned}
$$

being $\hat{x}$ and $\hat{S}$ the estimation vectors of state and output, respectively, and $L$ the observer gain matrix.

Controller module: Given the nature of the optimization problem, which is of mixed-integer linear programming nature, and the need to solve this problem fast enough to react in real time, it was chosen the solver IBM ILOG CPLEX Optimization Studio [27]. The simulations were developed in Matlabß using YALMIP toolbox [28] for stating the problem optimization in an intuitive format.

\section{Case study}

A test bench has been built to emulate the energy consumption behavior of a manufacturing machine and its peripheral devices with the aim to extract data and validate the proposed control strategy. Different types of loads were considered to emulate the real power consumption of both manufacturing and peripheral devices. In this sense, the test bench is composed of a three-phase delta connection motor, a heater, two Uninterruptible Power Supply (UPS) devices with different elements connected like loads (e.g., fans, lamps), and safety elements (e.g., regulators, relay). Moreover, a set of electronic devices, a PC, 
and a development board are included to control relays, allowing either activation or deactivation of the test-bench components according to an activation sequence sent. Besides, a data acquisition module with a sampling rate up to $250 \mu$ s is included for taking data and sent to both development board and central $\mathrm{PC}$, which receive the power signals $\left(S_{A}, S_{B}, S_{C}\right.$ [VA] ) pursuant to the activation sequence sent to test bench. An electrical diagram of the test bench with the connected loads is shown in Figure 3.

Due to the periodic behavior of considered systems, the testbench components were classified as peripheral and manufacturing devices. In this case, a operation cycle with two stages of manufacturing (cutting, milling, drilling, forming, etc.), was taken as the reference to represent the manufacturing sequence in the test bench. Thus, the heater and a UPS with a lamp and a fan connected were selected to construct the periodic sequence since that selection allows modulating loads to obtain the manufacturing stages. On the other hand, the available motor $\left(P_{1}\right)$ and a UPS with two fans connected $\left(P_{2}\right)$ were considered as the peripheral devices since they usually produce instantaneous peaks when are activated.

According to the previous discussion, a sequence $\boldsymbol{\Lambda}_{\mathbf{M}}$ was created with $T=29 \mathrm{~s}$, which is constant along the time. This latter fact does not hold for peripheral devices, which will be activated depending on both the global $S$ and the $q$-relations between each device and $\boldsymbol{\Lambda}_{\mathbf{M}}$. Next, different tests were performed in order to determine the most suitable sampling rate for taking the data to be used in the model identification. Thus, a sampling time of $\tau_{s}=10 \mathrm{~ms}$ was selected based on a trade-off between the temporal resolution of the signals and the computational time for running the proposed control design. In Figure 4 , the proposed manufacturing sequence and the energy consumption profiles of peripheral devices for only one phase are presented, as it is developed in most of reported works [29, 30].

Once $\boldsymbol{\Lambda}_{\mathbf{M}}$ and its energy consumption $\bar{\beta}$ are defined, the $q$ relations in (4) should be established taking into account the real operation of the peripheral devices, their operating ranges and their relations with $\boldsymbol{\Lambda}_{\mathbf{M}}$. It should be noted that, since most of the components are single-phase, the proposed approach will be developed considering only one phase $(B)$. However, all the analyses proposed in this paper can be properly extended for handling devices connected to all phases.

\section{1. q-relations}

According to Section 3.2, two situations were established to represent the real operation of two peripheral devices by using one motor $\left(P_{1}\right)$ and one UPS $\left(P_{2}\right)$. The considered situations are the following:

\subsubsection{Air-supply pump $\left(P_{1}\right)$}

One of the functions of pneumatic systems in manufacturing systems is to supply air of pre-defined conditions of pressure to clamp/unclamp pieces during manufacturing operations. Thus, considering a supply system as shown in Figure 5a, a pump provides to an air stream the energy enough to achieve the desired conditions of pressure, and then, this stream is transported towards the devices that required it. Due to the process performed, this supply system is considered as critical, with buffer capacity, and coupled to the manufacturing process.

Thus, the dynamic for the total change of mass $M_{T_{2}}$ and pressure $P_{T_{2}}$ inside the thank $T_{2}$ can be expressed in the discretetime version based on Taylor's series expansions and the finite difference discretization scheme as follows:

$$
M_{T_{2}}(k+1)=M_{T_{2}}(k)+\tau_{s} \sigma(k),
$$

being $\tau_{s}$ the sampling time, and $\sigma(k)=m_{\text {in,a }}(k)-m_{\text {out }, a}(k)$. On the other hand, the changes in pressure are related to changes in mass according to

$$
P_{T_{2}}(k)=\frac{M_{T_{2}}(k) R T}{V_{T_{2}} W_{M}},
$$

being $m_{\text {out }, a}$ the constant consumption of air from manufacturing system o device along $T, m_{\text {in, }}$ the air flow pumping from $P_{2}$ to $T_{2}$, and, $R, T, V_{T_{2}}, W_{M}$ and $n$ the gas constant, temperature, volume of $T_{2}$, the molecular weight and the moles of gas, respectively.

According to (11) and (12), the $q$-relations for $P_{2}$ and $\boldsymbol{\Lambda}_{\mathbf{M}}$ are defined. However, considering that $P_{T_{2}}$ is the variable to be monitored, these equations must satisfy the operating range of pressure $\underline{P}_{T_{2}} \leq P_{T_{2}}(k) \leq \bar{P}_{T_{2}}$, with $\underline{P}_{T_{2}}$ and $\bar{P}_{T_{2}}$ the lower and upper bounds of $P_{T_{2}}$, respectively.

\subsubsection{Coolant-supply pump $\left(P_{2}\right)$}

Similar to the air supply, the supply of coolant could be a critical task for some manufacturing processes (e.g., machining) because if the coolant is not supplied in either sufficient quantity or at suitable time instants, manufacturing processes will not correctly work and the desired properties of the piece will not be reached. In many cases, coolant-supply systems are designed with re-circulation, filtering and re-use of coolant, as shown in Figure $5 b$.

Based on Figure 5b, a constant flow of coolant $m_{c, 3}$ is pumped by $P_{3}$ from $T_{4}$ towards a clean-coolant tank $T_{3}$ passing through a filter in which the fine particles are separated. Afterwards, the coolant required for the different manufacturing devices (M1 and M2) is taken from $T_{3}$. Next, after manufacturing operation, the dirty coolant is collected and delivered to a dirty tank $T_{4}$ where gross chip particles are separated by gravity, and the process is repeated. In this case, the pump $P_{3}$ corresponds to the peripheral device of interest, which must be activated in order to supply the coolant flow required to guarantee both the manufacturing process and the reference levels at each tank. Therefore, in this case both the activation instant and the suitable flow of coolant to satisfy the operating constraints must be selected with $u_{P, 3} \in \mathbb{Z}_{\geq 0}$ such that $\underline{u}_{P, 3} \leq u_{P, 3} \leq \bar{u}_{P, 3}$, being $\underline{u}_{P, 3}$ and $\bar{u}_{P, 3}$ the lower and upper bounds of $u_{P, 3}$, respectively.

In this case, the level dynamics at each tank are the following:

- Clean tank $T_{3}$

$$
L_{c}(k+1)=L_{c}(k)+\tau_{s} \gamma(k)\left(\frac{1}{\rho_{c} A_{T_{3}}}\right),
$$

- Dirty tank $T_{4}$

$$
L_{d}(k+1)=L_{d}(k)+\tau_{s} \theta(k)\left(\frac{1}{\rho_{c} A_{T_{4}}}\right),
$$




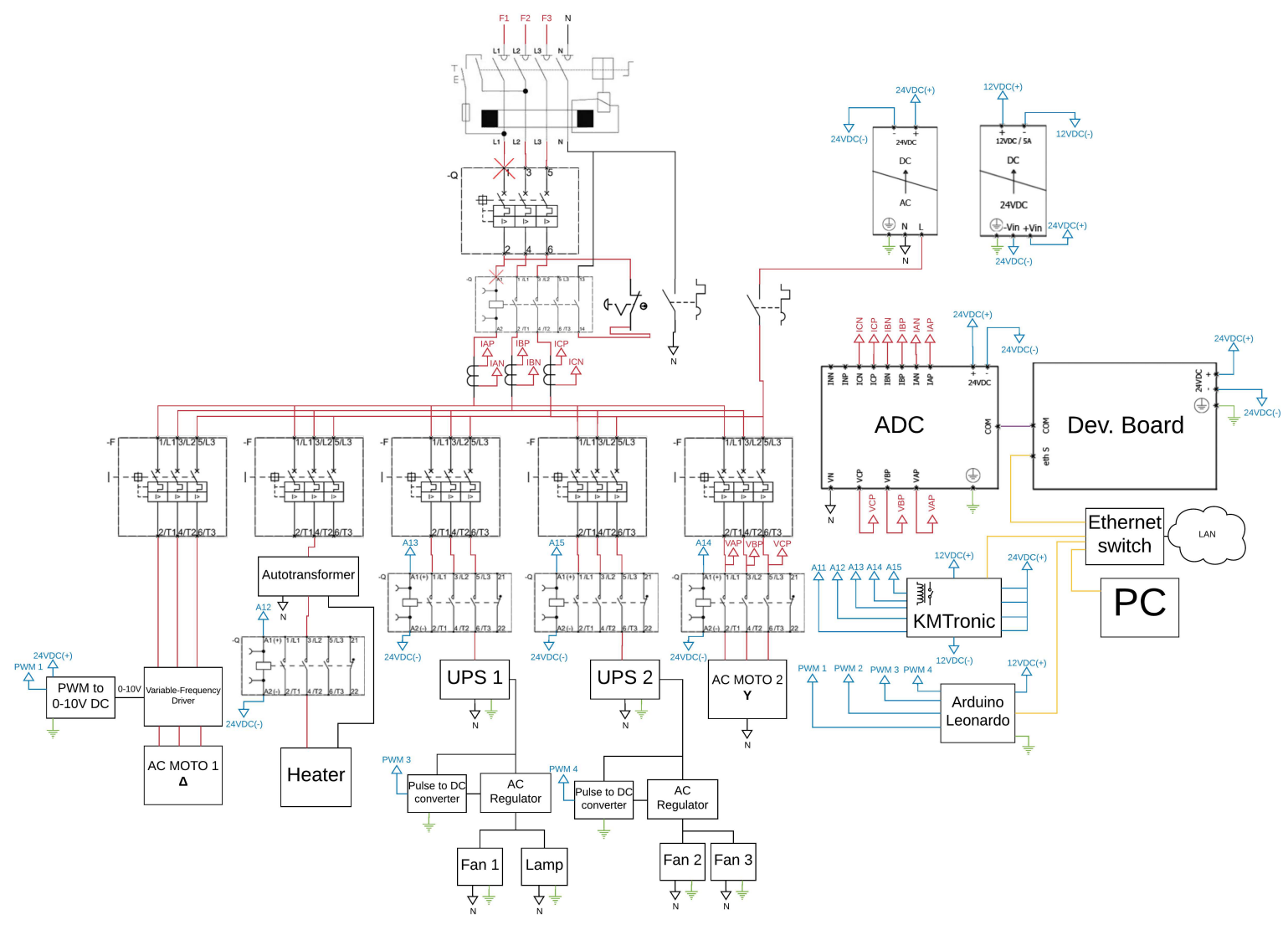

Figure 3: Electrical diagram of test bench of a machine tool emulator.

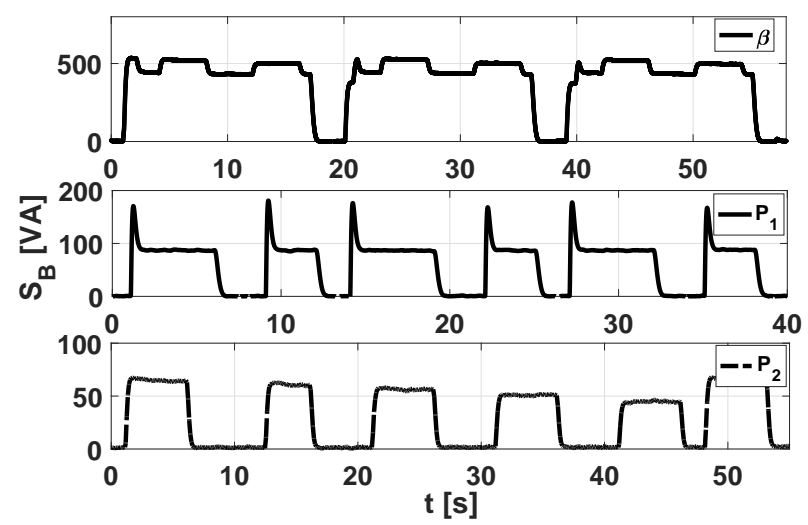

Figure 4: Energy consumption profile of (a) manufacturing sequence, (b) motor start connection $P_{1}$, and (c) UPS with fans $P_{2}$, for phase $B$.

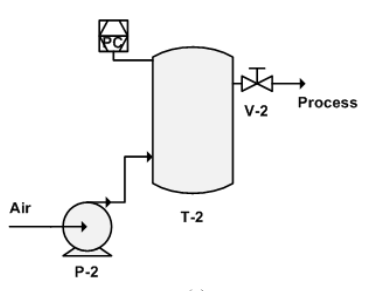

(a)

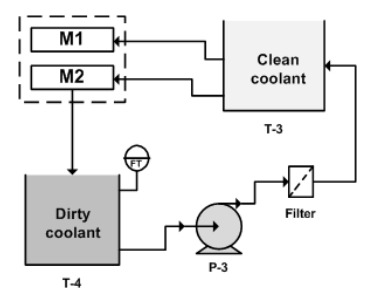

(b)
Figure 5: (a) Scheme of air supply to manufacturing process and (b) scheme of coolant supply to manufacturing process. with $\gamma(k)=m_{c, 3}(k)-m_{M 1,3}(k)-m_{M 2,3}(k)$ and $\theta(k)=m_{M 1,4}(k)+$ $m_{M 2,4}(k)-m_{c, 3}(k)$, where $m_{M 1,3}=h_{1}\left(\boldsymbol{\Lambda}_{\mathbf{M}}\right)$ and $m_{M 2,3}=h_{2}\left(\boldsymbol{\Lambda}_{\mathbf{M}}\right)$ are the coolant flows required for two manufacturing devices during manufacturing processes, whereas, $m_{M 1,4} \approx m_{M 1,3}$ and $m_{M 2,4} \approx m_{M 2,3}$ are the flows of dirty coolant from manufacturing devices to $T_{4}$. This approximation is due to the coolant losses during the recovery process.

Then, the coolant flow to be cleaned $m_{c, 3}$ is determined based on

$$
P_{\text {out }, 3}(k)+\eta \omega(k)=P_{\text {in }, 3}(k)+\rho_{c} h_{f_{1 \rightarrow 2}}(k),
$$

being $P_{\text {in, } 3}, P_{\text {out }, 3}, \rho_{c}, \eta, \omega$ and $h_{f_{1 \rightarrow 2}}$ the input pressure, output pressure, coolant density, efficiency of pump, specific work per time unit and the energy losses by friction, respectively. Taking into account the considerations reported in Appendix A, where this procedure is explained in detail, $m_{c, 3}$ should be determined taking into account the operating ranges $\underline{L}_{T_{3}} \leq L_{T_{3}} \leq \bar{L}_{T_{3}}$ and $\underline{L}_{T_{4}} \leq L_{T_{4}} \leq \bar{L}_{T_{4}}$, being $\underline{L}_{T_{i}}$ and $\bar{L}_{T_{i}}$ the lower and upper bounds for the level in the tank $T_{i}$, respectively, and the following expression for specific work $\omega$ :

$$
\omega(k)=\frac{W(k)}{m_{c, 3}(k)},
$$

being $W$ the work supply to the pump. Besides, in this case in which $u_{P_{3}} \in \mathbb{Z}$, the operating range of $W$ should correspond to the operating range of $m_{c, 3}$, i.e., $\underline{m}_{c, 3} \leq m_{c, 3}(k) \leq \bar{m}_{c, 3}$, with $\underline{m}_{c, 3}$ and $\bar{m}_{c, 3}$ the lower and upper bounds for coolant flow, respectively. Thus, based on (13) - (16), the activation instants and the 

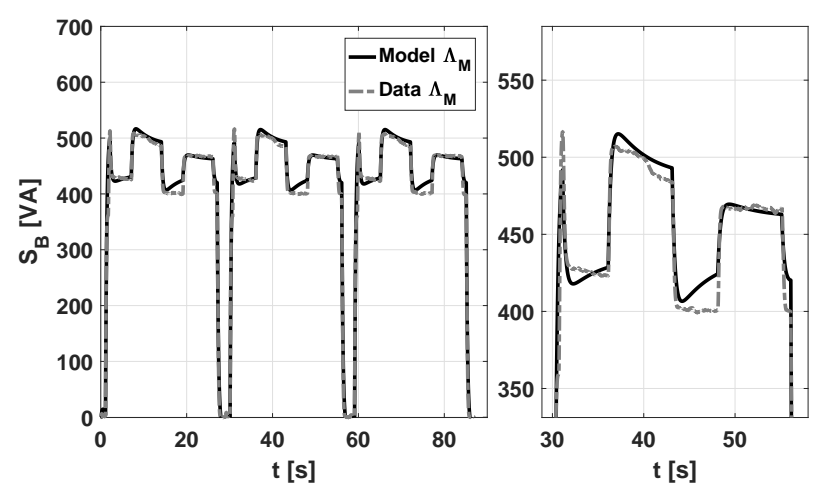

Figure 6: Validation of the manufacturing sequence model.

Table 1: Model order and fitting percentage between real and modeled output.

\begin{tabular}{|c|c|c|c|c|}
\hline Component & $\mathbf{P}_{\mathbf{1}}$ & $\mathbf{P}_{\mathbf{2}}$ & $\mathbf{P}_{\mathbf{3}}$ & $\boldsymbol{\Lambda}_{\mathbf{M}}$ \\
\hline \% fitting & 92.33 & 90.39 & 80.04 & 90.70 \\
\hline
\end{tabular}

coolant flow could be determined to satisfy the operating levels at each tank.

\section{Experimental results}

Based on the proposed approach in Section 3, the obtained results for model identification and the proposed control strategy are presented below.

\subsection{Model identification}

In order to obtain a state-space representation of energy consumption models as shown in (8), input-output data have been taken from the test bench for both manufacturing and peripheral devices according to the classification proposed in Section 4. Different sequences of $\boldsymbol{\Lambda}_{\mathbf{P}}$ and $\boldsymbol{\Lambda}_{\mathbf{M}}$ were tested to obtain the corresponding outputs $S_{P}[\mathrm{VA}]$ and $S_{M}$ [VA]. Afterwards, based on SI methods, energy consumption models were identified by using the routine n4sid of the System Identification Toolbox ${ }^{\mathrm{TM}}$ provided by Matlab $\AA$.

Based on the obtained data sets, different values of $N$ were tested in System Identification Toolbox ${ }^{\mathrm{TM}}$ with the aim to identify the suitable matrices $A, B, C$, and $D$, which allow the highest fitting degree between the real and modeled outputs. This procedure was performed for each one of the peripheral devices and the designed periodic sequence, considering the three phases of the test bench. However, since all devices were connected to the phase $B$, only the corresponding results to this phase will be presented.

In Figures 6 and 7 the validations of the obtained models for both the designed $\boldsymbol{\Lambda}_{\mathbf{M}}$ and peripheral devices are shown, respectively. In addition, in Table 1 the fitting percentages of each model output with respect to the available real data for a selected model order $N=3$ are presented. From these results, it is possible to observe that identified models are able to represent the dynamic behavior of both peripheral devices and the proposed manufacturing sequence with high precision and fitting values higher than $80 \%$. Thus, taking into account the optimization problem in (7), the energy consumption models, and the test bench, next, the obtained results for implementation of the proposed control strategy are exposed.

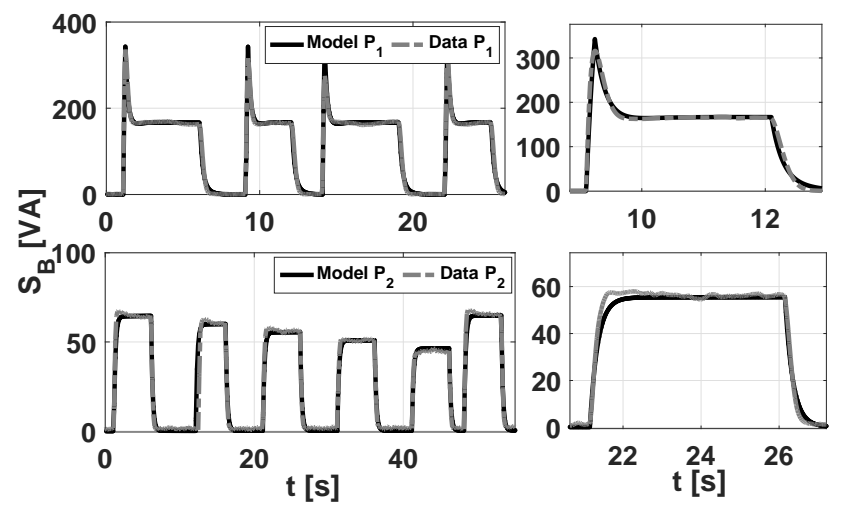

Figure 7: Validation of energy consumption models of peripheral devices $\left(P_{j}\right)$.

Table 2: Simulation parameters for peripheral devices.

\begin{tabular}{|c|c|c|c|}
\hline Parameter & Value & Parameter & Value \\
\hline$V_{T_{2}}$ & $0.005 \mathrm{~m}^{3}$ & $A_{T_{3}}$ & $0.0314 \mathrm{~m}^{2}$ \\
\hline$m_{\text {out }, a}$ & $0.0015 \frac{\mathrm{m}^{3}}{\mathrm{~s}}$ & $A_{T_{4}}$ & $0.0314 \mathrm{~m}^{2}$ \\
\hline$m_{\text {in, } a}$ & $0.0025 \frac{\mathrm{m}^{3}}{\mathrm{~s}}$ & $\eta$ & 0.85 \\
\hline$T$ & $25^{\circ} \mathrm{C}$ & $m_{t, 3}$ & $0.005 \frac{\mathrm{m}^{3}}{\mathrm{~s}}$ \\
\hline $\bar{P}_{T_{2}}$ & $6.5 \mathrm{bar}$ & $m_{w p, 3}$ & $0.006 \frac{\mathrm{m}^{3}}{\mathrm{~s}}$ \\
\hline$\underline{P}_{T_{2}}$ & $5 \mathrm{bar}$ & $h_{1 \rightarrow 2}$ & $0.06 \frac{\mathrm{m}^{2}}{\mathrm{~s}^{2}}$ \\
\hline$P_{\text {atm }}$ & $101325 \mathrm{~Pa}$ & $\Delta P_{\text {filter }}$ & $10000 \mathrm{~Pa}$ \\
\hline$\rho_{c}$ & $1042.5 \frac{\mathrm{kg}}{\mathrm{m}^{3}}$ & $\bar{L}_{T_{3}}$ & $0.5 \mathrm{~m}$ \\
\hline $\bar{L}_{T_{4}}$ & $0.8 \mathrm{~m}$ & $\underline{L}_{T_{3}}$ & $0.3 \mathrm{~m}$ \\
\hline$\underline{L}_{T_{4}}$ & $0.6 \mathrm{~m}$ & $R$ & $8.1314 \frac{\mathrm{J}}{\mathrm{Kmol}}$ \\
\hline
\end{tabular}

\subsection{Test-bench implementation}

Given the mixed-integer linear programming nature of the proposed optimization problem in (7) and the tools considered to solve it, preliminary simulations were developed in Matlab $(\mathbb{R}$ to test the performance of the proposed predictive-like controller before its implementation in the test bench. Thus, according to the proposed control strategy, both the simulations and the test bench implementation were performed according to the parameters in Table 2 for the operation of the peripheral devices.

It should be noted that, although the tests performed in the test bench were developed using a sampling time of $\tau_{S}=10 \mathrm{~ms}$, the proposed controller is executed at each second, finding optimal values of $u_{P_{j}}$ at each second and keeping this values up to the next second.

In addition to the proposed controller (OBC), a Rule-Based Control (RBC) scheme, in which the peripheral devices are turned on/off according to the limit values for $q$-relations, was tested and compared to the proposed approach. Thereby, every time that the minimum allowed value for a peripheral device is reached, this device is turned on, whereas if the maximum allowed value is achieved the device will be turned off. Besides, in order to avoid damages in the peripheral devices by a high-switching frequency that directly affects the inertia of each device, safety time constraints were considered into the optimization problem. Thus, at each second in which some pe- 
ripheral device is turned on/off, it should remain on/off during a time period $t_{S a f}=5 \mathrm{~s}$.

Finally, the proposed OBC, RBC and the observer were implemented in an embedded system using the $\mathrm{C}++$ language and the Basic Linear Algebra Subprograms (BLAS)[31] and Linear Algebra Packet (LAPACK)[32] libraries, in order to perform linear algebra operations in parallel to reduce as much as possible the computational time. The optimization problem (7) was implemented in ILOG CPLEX C++ API[27] that is an interface to use the CPLEX solver.

\subsection{Key Performance Indicators (KPI)}

In order to evaluate the energy efficiency and performance of the considered control strategies, the following KPIs were selected:

\subsubsection{Maximum peak}

It is important to consider the supply capacity or availability of electrical power when contracting with an energy company. It seeks to reduce the maximum monthly demand to reduce costs and contract less supply capacity, since exceeding this limit may result in economic penalties. Then, the maximum consumption or peak value for a test is expressed as

$$
\mathbf{K P I} \mathbf{I}_{1}=\|\mathbf{S}\|_{\infty},
$$

where $\mathbf{S}=\left\{S(0), S(1), \ldots, S\left(N_{\mathbf{S}}\right)\right\} \in \mathbb{R}_{\geq 0}$ is a set of measurements for some tests and, $N_{\mathbf{S}}=|\mathbf{S}|=\frac{H_{S}}{\tau_{s}} \in \mathbb{R}_{\geq 0}$ is the number of the measurements.

\subsubsection{Load Factor}

In order to assess whether the contracted supply capacity is appropriate, a suitable indicator is the load factor. It is defined as a ratio of the average load during a given period and the maximum demand in the same period, i.e., the amount of energy used with respect to the maximum capacity in a certain period of time (e.g., month, days, hours). Thus, the load factor is then defined as follows:

$$
\mathbf{K} \mathbf{I I}_{2}=\frac{1}{N_{\mathbf{S}} \mathbf{K} \mathbf{P I}} \sum_{k=1}^{H_{s}} S(k),
$$

being $\mathbf{K P I _ { 2 }}$ the load factor percentage derived by the average energy along $H_{s}$ regarding to the equivalent energy consumption at maximum load $\left(N_{\mathbf{S}} \mathbf{K} \mathbf{P I} \mathbf{I}_{1}\right)$. Thus, if the percentage is greater than $50 \%$, the use of energy is relatively constant and the contracted capacity is then properly used. Otherwise, there is a high demand for energy that might be reduced.

\subsubsection{Variance of energy consumption profile}

The management of the peripheral devices in an intelligent way to have an approximately constant consumption can be reached avoiding the sum of several power peaks and turning on the peripheral devices when the manufacturing process shows a low consumption. Thus, achieving an efficient distribution of energy over time with a low variation, the adequate supply capacity to be contracted could be determined. In this sense, the variance of energy consumption is defined as follows:

$$
\mathbf{K} \mathbf{P I}_{3}=\frac{1}{N_{\mathbf{S}}} \sum_{k=1}^{H_{s}}(S(k)-\overline{\mathbf{S}})^{2},
$$

being $\overline{\mathbf{S}}$ the mean value of energy consumption along $H_{s}$.
Table 3: KPI values for the OBC and RBC controllers.

\begin{tabular}{|c|c|c|c|}
\hline Controller $\backslash$ KPI & $\mathbf{K P I}_{1}$ & $\mathbf{K P I}_{2}$ & $\mathbf{K P I}_{3}$ \\
\hline $\mathrm{OBC}\left(H_{p}=T\right)$ & $831.6273 \mathrm{VA}$ & $60.53 \%$ & $13485 \mathrm{VA}$ \\
\hline $\mathrm{OBC}\left(H_{p}=2 T\right)$ & $831.6273 \mathrm{VA}$ & $60.53 \%$ & $13492 \mathrm{VA}$ \\
\hline $\mathrm{OBC}\left(H_{p}=3 T\right)$ & $831.6273 \mathrm{VA}$ & $60.53 \%$ & $13508 \mathrm{VA}$ \\
\hline $\mathrm{RBC}$ & $911.1758 \mathrm{VA}$ & $55.09 \%$ & $29328 \mathrm{VA}$ \\
\hline
\end{tabular}

Table 4: Improvement in KPI values of OBC with respect to $\mathrm{RBC}$ in the test bench.

\begin{tabular}{|c|c|c|c|}
\hline Controller $\backslash$ KPI & KPI $_{1}$ & $\mathbf{K P I}_{2}$ & $\mathbf{K P I}_{3}$ \\
\hline $\mathrm{RBC}\left(H_{p}=T\right)$ & $899.00 \mathrm{VA}$ & $66.66 \%$ & $30948 \mathrm{VA}$ \\
\hline $\mathrm{OBC}\left(H_{p}=T\right)$ & $830.37 \mathrm{VA}$ & $70.25 \%$ & $16466 \mathrm{VA}$ \\
\hline$\%$ improvement & $7.63 \%$ & $5.40 \%$ & $46.79 \%$ \\
\hline
\end{tabular}

\subsection{Results}

Once the initial conditions of the observer and the $q_{r^{-}}$ relations are established, four tests were carried out to compare both control strategies and determine the performance of OBC at different $H_{p}$. One test was performed using the RBC while the other three tests were executed considering the $\mathrm{OBC}$ for different values of $H_{p}$, i.e., $H_{p}=T, H_{p}=2 T$ and $H_{p}=3 T$. Each test was executed 10 times with a duration of 41 minutes, which corresponds to 86 operation cycles. Based on these tests, the proposed KPIs were calculated and the obtained results are presented in Table 3. Thus, according to the obtained results, $H_{p}=T$ was established for testing the control strategy in the test bench since increasing it does not bring great benefits in KPIs.

Next, in Table 4, a comparative of KPIs values for the implementation of the proposed OBC and RBC is shown as the improvement in the percentage of the OBC with respect to the RBC. It should be noted that since $\boldsymbol{\Lambda}_{\mathbf{M}}$ is not modified, i.e., the total time for manufacturing a piece is the same, the productivity of the system is not affected when the proposed control strategy is implemented. This fact is a consequence of the independent management of peripheral devices regarding global energy consumption in which the dynamics of these devices are considered as restrictions into the optimization problem. From this approach, it is possible to guarantee that peripheral devices are energy-efficient managed in a way in which the resources required for the machine operation are supplied in quantity and at the proper time instant. Based on the obtained results, it is possible to see that regarding $\mathbf{K P I}_{\mathbf{1}}$, the peak using $\mathrm{OBC}$ is reduced by $7.63 \%$ with respect to $\mathrm{RBC}$, while for $\mathbf{K P I}_{2}$ improvements close to $5.40 \%$ were achieved. In this regard and according to the total simulation time, 86 pieces were produced when both $\mathrm{OBC}$ and the conventional RBC are implemented. Therefore, the proposed OBC improves energy efficiency without productivity losses. On the other hand, the variation of the energy consumption profile is reduced by $46.79 \%$, which means a more constant energy consumption profile can be reached using the proposed controller while the system productivity is remained.

Afterward, in Figure 8 a representative part of the energy consumption profile resulting of application of the $\mathrm{OBC}$ and RBC controllers is presented. Based on these results, it observes that power peaks obtained from RBC are always higher 


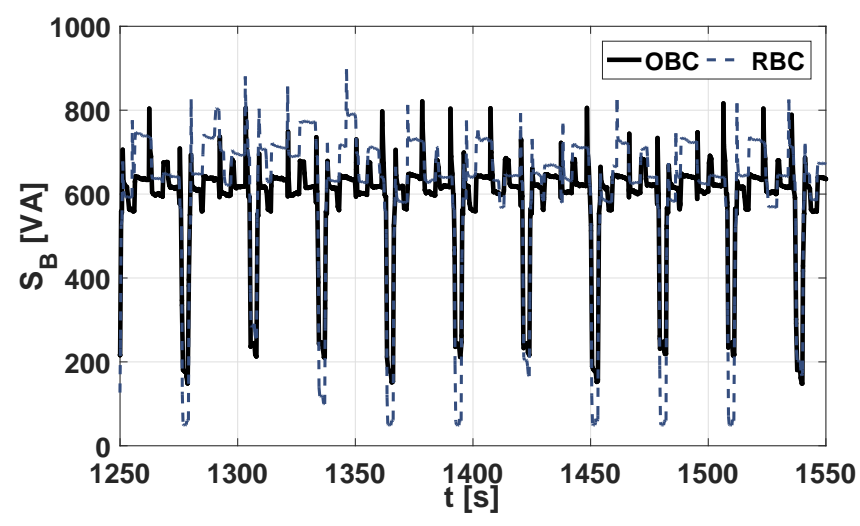

Figure 8: A zoom of energy consumption profile for both OBC and RBC.

than peaks from the OBC strategy, in concordance with results in Table 3. This behavior is mainly due to the RBC does not take into account the current stage of the manufacturing process for taking a decision of switching on/off any peripheral devices. Besides, the proposed OBC is able to modulate the load of $P_{2}$ in order to satisfy operating constraints and reach a lower energy consumption.

Representative parts of both the optimal input sequence $\Gamma^{*}$ for $\mathrm{OBC}$ and the activation/deactivation sequence for $\mathrm{RBC}$ are presented in Figure 9. From these results, it is possible to see that even when the safety constraints are satisfied, the switching frequency of $\mathrm{OBC}$ is higher than the one of the $\mathrm{RBC}$ for both peripheral devices. Thus, the OBC strategy avoids to turn devices on at the same time instant, and in the cases that both devices must switch on the activation of one of them is delayed or advanced to avoid their simultaneous activation. Besides, when the load can be modulated (like for $P_{2}$ ), OBC never decides to turn devices on at the maximum capacity and instead of that, it increases the switching frequency at lower consumption levels.

On the other hand, the dynamics of $q_{r}$-relations for the two processes selected and depicted by peripheral devices are shown in Figure 10. From these results, and according to the optimal sequences found, it can see that for both cases the operation of peripheral devices remains inside the considered operating ranges. However, for the case of the coolant-supply system, which shows the slowest dynamics, $P_{2}$ is activated in a manner in which the level of the clean tank keeps near the lower limit while most of the recovered coolant is stored into the dirty tank, i.e., it remains near upper bound as shown in Figure 11. This behavior could be a consequence of energy losses considered at each section of the process in Figure 5 (e.g. from $M 1, M 2$ to $T_{4}$ and from $T_{4}$ to $T_{3}$ ), since the system requires more energy to transport fluid through the filter and pipeline up to $T_{3}$. Therefore, the OBC takes the decision of storing the coolant in the dirty tank and, only when this is required, the coolant is pumped towards the filter and clean tank.

\section{Conclusion}

Based on the obtained results, it is possible to conclude that the $\mathrm{OBC}$ manages peripheral devices to find an efficient

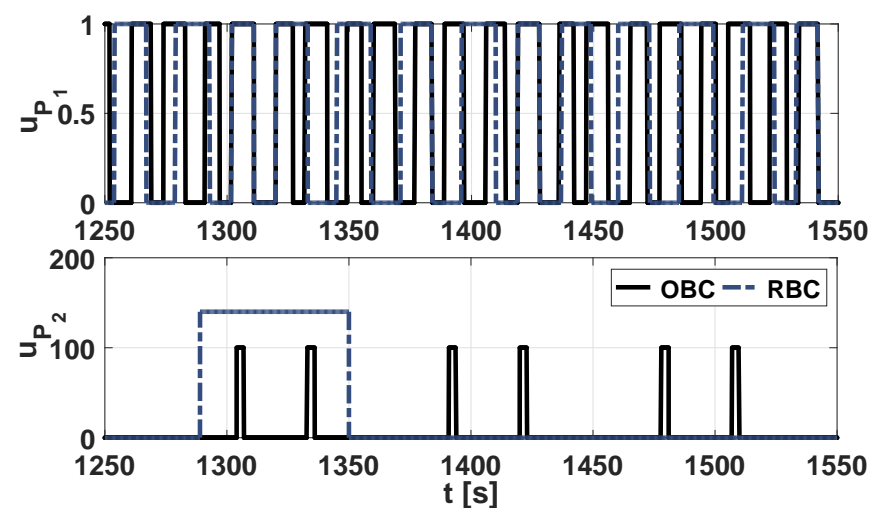

Figure 9: Input signals for the peripheral devices.

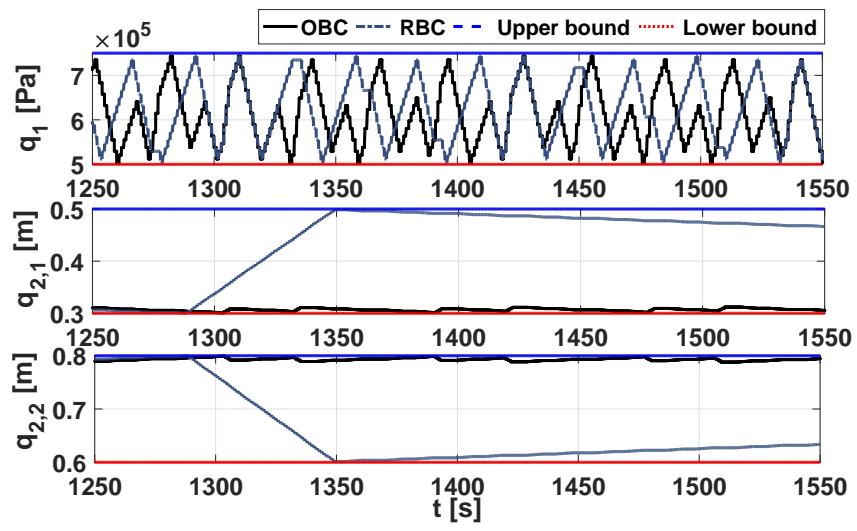

Figure 10: Dynamics of $q$-relations for $P_{1}$ and $P_{2}$.

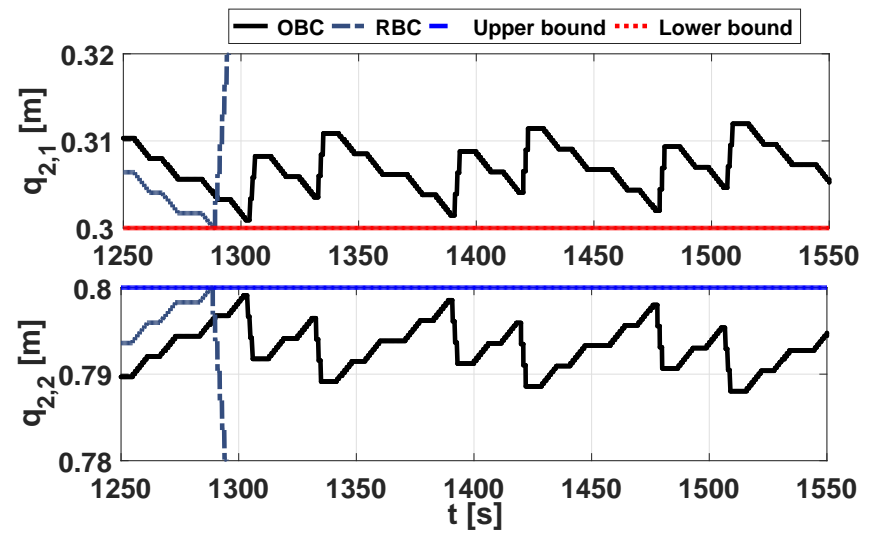

Figure 11: Zoom of $q$-relations dynamics for $P_{1}$ and $P_{2}$ presented in Figure 10.

way to activate/deactivate them, taking into account the periodic behavior of the manufacturing process. Thus, according to the considered KPIs, the proposed control strategy allows achieving reductions in energy costs avoiding the economic penalties produced by surpassing the nominal power purchased. Therefore, based on the proposed control strategy based on OBC techniques, in which the problem formulation can be customized including both the operating constraints and existent relations between the manufacturing system and its peripheral devices, these latter can be correctly managed regarding energy efficiency without compromising the normal operation and productivity of the manufacturing processes.

In addition, it should be noted that the use of loads that can be 
modulated could represent a energy-saving opportunity since in this way it is possible to modify its energy consumption without change the physical configuration of the peripheral devices. However, although the OBC techniques have great advantages, the inclusion of a lot of peripheral devices in which both the activation instants and the optimal value should be determined, besides their operational constraints, could make the optimization problem more complex. This fact may produce that the computing times increase, and therefore, the use of Mixed Logical Dynamical (MLD) systems could be an efficient way to model these systems at machine level for its posterior application at line or plant levels.

Finally, although the proposed approach can achieve an optimal solution considering the operating limitations, the input delays, and the time synchronization to close the loop, a stage of co-design to implement some elements in a FieldProgrammable Gate Array (FPGA) should consider as a first approximation to test the proposed control strategy in a real manufacturing system.

\section{Acknowledgements}

This work has been funded by the project IKERCON (ref. C10683) and partially supported by the Spanish State Research Agency through the María de Maeztu Seal of Excellence to IRI (MDM-2016-0656). Jenny Díaz has been supported by FIAGAUR scholarship programmee of the Catalan Government.

\section{References}

[1] IEA, International energy agency: World energy statistics 2017, urlhttp://www.iea.org/statistics/ (2018).

[2] S. Bilgen, Structure and environmental impact of global energy consumption, Renewable and Sustainable Energy Reviews 38 (2014) 890-902. doi:10.1016/j.rser.2014.07.004.

[3] G. Ingarao, Manufacturing strategies for efficiency in energy and resources use: The role of metal shaping processes, Journal of Cleaner Production 142 (2017) 2872-2886. doi:10.1016/j.jclepro.2016.10. 182.

[4] L. Zhou, J. Li, F. Li, Q. Meng, J. Li, X. Xu, Energy consumption model and energy efficiency of machine tools: a comprehensive literature review, Journal of Cleaner Production 112 (Part 5) (2016) 3721-3734. doi: 10 . 1016/j.jclepro.2015.05.093.

[5] J. Duflou, J. Sutherland, D. Dornfeld, C. Herrmann, J. Jeswiet, S. Kara, M. Hauschild, K. Kellens, Towards energy and resource efficient manufacturing: A processes and systems approach, CIRP Annals - Manufacturing Technology 61 (2) (2012) 587-609. doi:10.1016/j.cirp.2012. 05.002 .

[6] A. Tivay, M. Zareinejad, S. Rezaei, K. Baghestan, A switched energy saving position controller for variable-pressure electro-hydraulic servo systems, ISA Transactions 53 (4) (2014) 1297-1306, disturbance Estimation and Mitigation. doi : 10.1016/j.isatra.2014.04.010.

[7] Z. Zhang, . Tang, T. Peng, L. Tao, S. Jia, A method for minimizing the energy consumption of machining system: integration of process planning and scheduling, Journal of Cleaner Production 137 (2016) 1647-1662. doi:10.1016/j.jclepro.2016.03.101.

[8] J. Yan, L. Li, F. Zhao, F. Zhang, Q. Zhao, A multi-level optimization approach for energy-efficient flexible flow shop scheduling, Journal of Cleaner Production 137 (2016) 1543-1552. doi :10.1016/j · jclepro. 2016.06.161.

[9] C. Lu, L. Gao, X. Li, Q. Pan, Q. Wang, Energy-efficient permutation flow shop scheduling problem using a hybrid multi-objective backtracking search algorithm, Journal of Cleaner Production 144 (2017) 228-238. doi:10.1016/j.jclepro.2017.01.011.
[10] S. Ubach, J. Diaz, C. Ocampo-Martinez, M. Antunez, Peak shaving through closed-loop optimization applied to machine tools with periodic behaviour, in: 2017 IEEE 3rd Colombian Conference on Automatic Control (CCAC), 2017, pp. 1-7. doi:10.1109/CCAC. 2017.8276433.

[11] R. Bhinge, J. Park, K. H. Law, D. A. Dornfeld, M. Helu, S. Rachuri, Toward a generalized energy prediction model for machine tools, Journal of Manufacturing Science and Engineering 139 (4) (2016) 1-12. doi : 10.1115/1.4034933.

[12] S. J. Qin, An overview of subspace identification, Computers \& Chemical Engineering 30 (10) (2006) 1502-1513, papers form Chemical Process Control VII. doi:10.1016/j.compchemeng . 2006.05.045.

[13] S. Zaiser, M. Buchholz, K. Dietmayer, Rotor temperature modeling of an induction motor using subspace identification, IFAC-PapersOnLine 48 (28) (2015) 847-852, 17th IFAC Symposium on System Identification SYSID 2015. doi:10.1016/j . if acol.2015.12.235.

[14] B. Sinquin, M. Verhaegen, A subspace like identification method for large-scale LTI dynamical systems, in: 2017 Signal Processing Symposium (SPSympo), 2017, pp. 1-6. doi : 10.1109/SPS . 2017.8053664.

[15] M. Yu, J. Liu, H. Wang, Nuclear norm subspace identification for continuous-time stochastic systems based on distribution theory method, ISA Transactions 83 (2018) 165-175. doi : 10.1016/j . isatra. 2018. 08.014.

[16] M. Kheradmandi, P. Mhaskar, Model predictive control with closed-loop re-identification, Computers \& Chemical Engineering 109 (2018) 249260. doi:10.1016/j.compchemeng. 2017.11.016.

[17] V. Meidanshahi, B. Corbett, T. A. Adams, P. Mhaskar, Subspace model identification and model predictive control based cost analysis of a semicontinuous distillation process, Computers \& Chemical Engineering 103 (2017) 39-57. doi : 10.1016/j . compchemeng. 2017.03.011.

[18] J. Maciejowski, Predictive Control with Constraints, Prentice Hall, Great Britain, 2002.

[19] J. Rawlings, D. Mayne, Model Predictive Control: Theory and Design, Nob Hill Publishing, Madison, WI (USA), 2009.

[20] C. Papadopoulos, J. Li, M. O'Kelly, A classification and review of timed markov models of manufacturing systems, Computers \& Industrial Engineering 128 (2019) 219-244. doi : 10.1016/j . cie.2018.12.019.

[21] A. Dideban, H. Zeraatkar, Petri net controller synthesis based on decomposed manufacturing models, ISA Transactions 77 (2018) 90-99. doi:10.1016/j.isatra.2018.04.008.

[22] Z. Fei, S. Li, Q. Chang, J. Wang, Y. Huang, Fuzzy petri net based intelligent machine operation of energy efficient manufacturing system, in: 2018 IEEE 14th International Conference on Automation Science and Engineering (CASE), 2018, pp. 1593-1598. doi : 10.1109/COASE. 2018. 8560366 .

[23] J. Wang, Y. Feng, Z. Fei, S. Li, Q. Chang, Markov chain based idle status control of stochastic machines for energy saving operation, in: 2017 13th IEEE Conference on Automation Science and Engineering (CASE), 2017, pp. 1019-1023. doi : 10.1109/COASE. 2017.8256236.

[24] P. Overschee, B. De Moor, Subspace Identification for Linear Systems : Theory - Implementation - Applications, Springer US, Boston, MA, 1996.

[25] M. Verhaegen, A. Hansson, N2sid: Nuclear norm subspace identification of innovation models, Automatica 72 (2016) 57-63. doi:10.1016/j. automatica.2016.05.021.

[26] B. De Moor, P. Van Overschee, W. Favoreel, Algorithms for Subspace State-Space System Identification: An Overview, Birkhäuser Boston, Boston, MA, 1999, pp. 247-311. doi: 10.1007/978-1-4612-0571-5_6.

[27] I. ILOG, Ibm ilog cplex optimization studio, v12. 5 (2013).

[28] J. Löfberg, Yalmip : A toolbox for modeling and optimization in Matlab, in: In Proceedings of the CACSD Conference, Taipei, Taiwan, 2004.

[29] W. Lee, S. H.K., J. Park, B. Min, Simulation-based machining condition optimization for machine tool energy consumption reduction, Journal of Cleaner Production 150 (2017) 352-360. doi:10.1016/j.jclepro. 2017.02 .178$.

[30] R. Popp, C. Liebl, M. Zaeh, Evaluation of the energy flexible operation of machine tool components, Procedia CIRP 63 (2017) 76-81. doi: 10. 1016/j.procir.2017.02.037.

[31] L. Blackford, A. Petitet, R. Pozo, K. Remington, R. Whaley, J. Demmel, J. Dongarra, I. Duff, S. Hammarling, G. Henry, An updated set of basic linear algebra subprograms BLAS, ACM Transactions on Mathematical Software 28 (2) (2002) 135-151. 
[32] E. Anderson, Z. Bai, J. Dongarra, A. Greenbaum, A. McKenney, J. Du Croz, S. Hammarling, J. Demmel, C. Bischof, D. Sorensen, LAPACK: A portable linear algebra library for high-performance computers, in: Proceedings of the 1990 ACM/IEEE conference on Supercomputing, IEEE Computer Society Press, 1990, pp. 2-11. 